\title{
Quick stable isotope analysis of multiple components in a complex mixture by Fourier transform mass spectrometry
}

\author{
SARAH ZEICHNER ${ }^{1 *}$, ELLE CHIMIAK ${ }^{1}$, JAMIE ELSILA ${ }^{2}$,
} JOHN EILER ${ }^{1}$

*corresponding author, szeichner@caltech.edu

${ }^{1}$ Division of Geological and Planetary Sciences, California Institute of Technology, Pasadena CA 91125

${ }^{2}$ Solar System Exploration Division, NASA Goddard Space Flight Center, Greenbelt, Maryland 20771

Recent developments in Fourier transform mass spectrometry facilitate high-resolution, high-precision measurements of molecular isotopic structure (i.e., sitespecific and multiple substitution), using a Thermo Scientific $^{\mathrm{TM}}$ Orbitrap ${ }^{\mathrm{TM}}$ mass analyzer coupled to Thermo Scientific Q Exactive $\mathrm{GC}^{\mathrm{TM}}$ and $\mathrm{Q}$ Exactive $\mathrm{HF}^{\mathrm{TM}}$ instruments. Precision of measurements on these instruments is constrained by the duration of eluted chromatographic peaks, shot-noise limits, and space charge effects within the Orbitrap, but can achieve $\pm 0.015 \%$ for long integration times $^{1}$. In this study, we observed natural abundance isotope ratios of molecular and fragment ions for a mixture of $\sim 10$ amino acids via direct injection of peaks eluted from the gas chromatograph $^{1}$, where each molecule in the complex mixture was analyzed for $\sim 30$ seconds. Altogether, direct analysis provides information on isotopic molecular structure for fragment ions of all eluted moleules in $\sim 40$ minutes (assuming appropriate standards in a standard mixture). Relative standard errors (RSE) for these measurements were as good as $33 \%$ for $\delta^{13} \mathrm{C}$ values of major fragment ions for one injection; averaging across multiple injections $(n=3)$ achieved RSE as good as 5.1\%. The direct injection method was designed to search for large site speicfic anomalies $(\sim 100 \%)$ in extraterrestrial compounds, but, at best performance, isotope effects on the order of $\sim 20 \%$ could be confidently recognized. To demonstrate the applicability of this method, we performed this direct analysis techinque on a mixture of amino acids extracted from the Murchison meteorite and identified site-specific ${ }^{13} \mathrm{C}$ enrichments ranging up to $120 \%$. These measurements provide information on precursor molecules in the solar system and extraterrestrial abiotic organic synthesis, and have implications for Early Earth origins of life chemistry.

\footnotetext{
1. Eiler, J. (Caltech) et al. Analysis of molecular isotopic structures at high precision and
}

accuracy. Int. J. Mass Spectrom. 422, 126-142 (2017). 